\title{
Validating a Standardized Approach to the Taylor Aggression Paradigm
}

\author{
in press at Social Psychological and Personality Science \\ David S. Chester ${ }^{\star}$ Emily N. Lasko \\ Department of Psychology, Virginia Commonwealth University, USA
}

Main Text Word Count: 4,893

${ }^{*}$ Correspondence should be addressed to:

David S. Chester

302 Thurston House

Virginia Commonwealth University

Richmond, VA, 23284, USA

dschester@vcu.edu

1-804-828-7624 


\begin{abstract}
The Taylor Aggression Paradigm (TAP) is a frequently-used laboratory measure of aggression. However, the flexibility inherent in its implementation and analysis can undermine its validity. To test whether the TAP is a valid aggression measure irrespective of this flexibility, we conducted two preregistered studies (Study $1 N=177$, Study $2 N=167$ ) of a standardized version of the TAP. Across both studies, TAP scores showed agreement with other laboratory aggression measures, were magnified by an experimental provocation manipulation, and were associated with traits typically linked to aggressive behavior. Mixed evidence was found for the external and discriminant validity of the task. Individual responses largely loaded onto a single component, suggesting that the aggregate scoring approach accurately represents the underlying data structure. These results provide preliminary support for the internal validity of this TAP approach and highlight the utility of preregistration in psychometric research.
\end{abstract}

Keywords: aggression, Taylor Aggression Paradigm, preregistration, validation, Competitive Reaction-Time Task 


\section{Introduction}

The study of aggressive behavior has historically been a challenging endeavor, entailing substantial ethical, logistical, and theoretical hurdles. The Taylor Aggression Paradigm (TAP; Taylor, 1967) arose from these early attempts at operationalization. Now past its $50^{\text {th }}$ year, the task has made great contributions to psychological science but has also received substantive criticism. In the present research, we employed preregistration to validate a single variant of the TAP in the absence of methodological and quantitative flexibility.

\section{The Taylor Aggression Paradigm: A Brief History and Overview}

As stated by Taylor, "In order to investigate aggression in the laboratory it is necessary to have an effective method for inciting aggression and for objectively measuring the aggressive responses that follow" (pp. 297, Epstein \& Taylor, 1967; Taylor 1967). To extend laboratory aggression measurement beyond hypotheticals, self-reports, and projective tests, Taylor simulated a provocative social experience in the lab and objectively measured aggressive responses. In the original TAP, participants selected the intensity or duration of an electric shock to administer to an opponent, who ostensibly did the same for them (Epstein \& Taylor, 1967; Shortell, Epstein, \& Taylor, 1970; Taylor, 1967). Participants then repeatedly competed against their opponent to flip a switch faster in response to a cue. If they lost the competition, participants received a shock of their opponent's choosing. Participants could see the level of the shock that their opponent selected for them, which allowed the experimenters to manipulate the level of provocation experienced by participants. 
Today, the TAP is most-frequently implemented as a computer program that can administer noise blasts through headphones instead of shocks delivered through electrodes (Bond \& Lader, 1986; Bushman, 1995). In this new approach, participants can set the duration and volume of these noise blasts and these settings serve as the laboratory operationalization of aggression. The TAP often includes multiple trials in order to provide a more reliable estimate of aggressive behavior. This task has gone on to serve as a mainstay aggression measure, even being modified for use in the MRI brain scanning environment to allow for the investigation of the neural basis of aggression (e.g., Chester \& DeWall, 2016). The original TAP publication (Taylor, 1967) has been used widely with 731 scholarly papers citing this paper from 1967-2017 (citation estimates from Google Scholar). However, such popularity has not been reached without criticism.

\section{Challenges and Critiques}

Laboratory measures of aggression, such as the TAP, have been categorically criticized for lacking many things (e.g., construct validity, ecological validity, experimental realism; Ferguson, Smith, Miller-Stratton, Fritz, \& Heinrich, 2008; Tedeschi \& Quigley, 1996, 2000). Rebuttals to these critiques abound, highlighting the TAP's convergent, external, and discriminant validity (Anderson \& Bushman, 1997; Giancola \& Parrott, 2008; Giancola \& Zeichner, 1995). More recently, the TAP has been criticized for a lack of standardization between and within laboratories (Elson, Mohseni, Breuer, Scharkow, \& Quandt, 2014). For instance, some studies only analyze volume or duration settings (not both) or the first or second trial (not all trials) of the task. The 
number of trials in the task can also vary widely. Such flexibility can be a strength, allowing researchers to tailor the task to a given study or hypothesis.

However, this methodological and quantitative flexibility can also be problematic if researchers test their hypotheses using multiple scoring and analytic strategies and then present the result that best fits their hypothesis as coming from a singular, preplanned test (Simmons, Nelson, \& Simonsohn, 2011). Such 'researcher degrees-offreedom' capitalize on chance and increase the likelihood of type 1 errors. Fortunately, preregistration provides a simple solution to ensure that flexible measures are not abused. Researchers publicly specify their hypotheses, methods, and planned analyses before data analysis begins, preventing any flexible and exploratory analyses from being reported as singular and confirmatory (Wagenmakers, Wetzels, Borsboom, van der Maas, \& Kievit, 2012). This preregistration technique can then be applied to validate the TAP, independent of such flexibility.

\section{Validation Approaches to the TAP}

There are many ways to demonstrate that the TAP is a valid and useful laboratory aggression measure. First, TAP scores should correspond to other measures of physically aggressive behavior and tendencies (e.g., laboratory aggression tasks, self-report aggression questionnaires), suggesting convergent validity of the task (Giancola \& Zeichner, 1995). Second, the TAP should be responsive to situational factors known to magnify aggressive behavior (e.g., provocation; Giancola \& Parrott, 2008), and correspond to personality traits that are reliably-linked to aggression (e.g., antagonism: Vize et al., in press), suggesting construct validity of the task. Third, variables that are distinct from physically harming others (e.g., verbally harming others, 
harming the self) should not correspond to TAP scores, thus demonstrating the task's discriminant validity (Bernstein, Richardson, \& Hammock, 1987). Fourth, as an index of the task's potential external validity, TAP scores should correspond to real-world acts of physical aggression (Anderson \& Bushman, 1997). If a preregistered version of the TAP is shown to exhibit these five qualities, then this task can be considered a valid aggression measurement, beyond concerns regarding the task's flexibility. To demonstrate the TAP's validity outside the context of methodological and quantitative flexibility, we conducted two preregistered studies. In both studies, a 25-trial version of the TAP was administered alongside variables that would provide support for the forms of validity detailed above.

\section{Study 1}

The preregistration plan for this study is available online (https://osf.io/x7rjb/register/565fb3678c5e4a66b5582f67), as are corresponding data, analysis code, and materials (https://osf.io/a2wft/files/).

\section{Methods}

\section{Participants}

A power-analysis using $\mathrm{G}^{*}$ Power 3.1 for an effect size of $r=.25, d=.47$ (an estimate based on mean TAP score correlations with trait physical aggression from Webster et al., 2014), an alpha level of .05 , and $90 \%$ power returned a planned sample size of 160. In an attempt to recruit at least 160 participants who did not meet exclusionary criteria, we recruited 211 undergraduate students from an introductory psychology subject pool, ceasing recruitment at the end of the academic year in which the study took place. Of these 211 participants, 25 were missing data necessary for our 
confirmatory analyses and 9 others expressed 'total disbelief' of our deception on our post-debriefing questionnaire. Final participants were therefore 177 undergraduates (68.4\% female, $31.6 \%$ male; Age: $M=20.01, S D=4.83$, range: $18-55)$. Participants' racial composition was 45.8\% White, 19.8\% African-American, 18.1\% Asian-American, and $16.4 \%$ Other. The sample was $13.0 \%$ Hispanic and $87.0 \%$ Non-Hispanic. Participants were compensated with course credit.

\section{Measures}

Taylor Aggression Paradigm. The version of the TAP used in both studies took the form of the computerized Competitive Reaction Time Measure of Aggression, version 2.9.9.9 for PC (Bushman \& Baumeister, 1998). For each of the 25 trials of the task, participants began by setting the volume (60-105 decibels, in 5 decibel increments) and duration ( $0-5$ seconds, in 0.5 second increments) of the noise blasts. Noise blast volumes were calibrated with a decibel reader to assure fidelity to the displayed decibel level. Participants could also set the volume or duration to 0 to prevent any noise blast from being administered. After participants entered their noise blast settings, they competed against their opponent (i.e., a same-sex VCU undergraduate) to press click a mouse button faster in response to a cue. If participants lost a given trial (which they did approximately $50 \%$ of the time), they were blasted with noise at the volume and duration that their opponent ostensibly determined ahead of time. Wins and losses were randomized across trials (yet this random order was held constant across participants). Participants' opponents began the task by selecting the highest noise settings. To ensure the task's realism, participants lost every trial that they 
did not respond to within two seconds. Further, trials would not advance if participants simply clicked the mouse repeatedly on the colored square.

Voodoo Doll Aggression Task. The VDT takes advantage of the innate human tendency to imbue certain objects with symbolic properties (Rozin, Millman, \& Nemeroff, 1986), asking participants to harm a representation of a real person (DeWall et al., 2013). In this task, participants were instructed to view a computer image of a plush human doll as a symbolic representation of their actual essay evaluator. Participants viewed what the doll would look like with an array of sharp pins stuck in it. Then, participants typed the number of virtual, sharp pins that they wished to stick into the doll (from 0 to 51 pins).

Brief Aggression Questionnaire. The 12-item BAQ is a subset of items from the 29-item Buss-Perry Aggression Questionnaire (Webster et al., 2014). The BAQ possesses a four factor structure with 3-item subscales measuring each construct: anger, hostility, physical aggression, and verbal aggression. Participants completed the full, 29-item Buss-Perry Aggression Questionnaire, and therefore the BAQ.

Aggressive Motives Scale. The 6-item AMS is a retrospective, self-report measure of whether participants' aggressive behavior on the TAP reflected instrumental or vengeful-harm motives (Anderson \& Murphy, 2003). This scale allowed us to disentangle whether participants acted aggressively on the TAP out of a simply competitive or truly aggressive motivation.

Non-Suicidal Self-Injury Assessment Tool. The 6-item NSSIAT measures state-level desires to engage in self-harm (Chester, Whitt, Davis, \& DeWall, 2017). 
History of Physical Fights Scale. This open-ended measure simply asked participants how many physical fights they had been in, in the past 5 years and the past year. This measure allowed for the assessment of real-world acts of aggressive behavior.

Provocation Manipulation Check. The 7-item PMC (Denson, von Hippel, Kemp, \& Teo, 2010) measured the extent to which the essay feedback provocation manipulation made participants feel provoked and insulted.

\section{Procedure}

Participants arrived individually to the laboratory, where they read and signed an informed consent form. Afterwards, participants were screened for sensitive hearing, due to the study's use of noise blasts. In order to experimentally-induce interpersonal provocation, this study used a validated essay evaluation paradigm in which participants received harsh or positive feedback on an essay (Bushman \& Baumeister, 1998;

Chester \& DeWall, 2017). The essay evaluation contained either negative (8/35 points, "One of the WORST essays l've EVER read!") or positive (33/35 points, "Great essay!") feedback, as determined by random assignment.

Laboratory aggression tasks. The experimenter then directed participants to the computer to complete the TAP, describing it as a measure of reaction time. The experimenter explained the task to the participant, gave them a sample of a moderately loud noise blast, and then left to check on the fictitious partner's internet connection to the participant. The experimenter returned quickly and instructed the participant to begin the task. After completing the TAP, the experimenter returned to the participant with a plate of crackers and a bottle of hot sauce to conduct the Hot Sauce Aggression Task 
(Lieberman, Solomon, Greenberg, \& McGregor, 1999). Participants were informed that they would now complete a measure of pain regulation, in which they would eat spicy hot sauce. After the participant added their desired level of hot sauce, the experimenter returned and placed a second plate of crackers in front of the participant and asked them if they would help by applying hot sauce to these crackers, which their essay evaluation partner would have to eat. After a 60 -second absence, the experimenter took both plates and weighed them away from the participant. Participants then completed the VDT and questionnaires.

Suspicion probe and debriefing. Participants completed a funneling suspicion interview, were debriefed, and retrospectively reported their level of suspicion during the study on a questionnaire.

\section{Results}

\section{Deviations from Preregistration Plan}

We were unable to achieve the $50 \%$ gender equity that we outlined in our preregistration plan and we did not enact the outlier exclusion rule, as this proved to be far too conservative and would have led to the exclusion of a substantial portion of our sample. We excluded participants who expressed total disbelief in our deception procedures, which was not outlined in our preregistration. Confidence intervals were calculated conventionally, rather than via nonparametric bootstrapping.

\section{Descriptive Statistics}

We scored the TAP by calculating the mean of all 50 individual measurements of the task ( 2 settings per trial $\times 25$ trials; Table 1 ). The voodoo doll pin count and number of physical fights were zero-inflated and positively-skewed (Table 1), which required 
statistical modeling that specified a Poisson distribution (as recommended by DeWall et al., 2013). Internal consistency was adequate for all self-report measures, except the Verbal subscale of the BAQ. Zero-order correlations between key study variables are summarized in Supplemental Table 1.

Table 1. Descriptive statistics for Study 1 confirmatory variables: Aggressive Motives Scale (AMS), Brief Aggression Questionnaire (BAQ), History of Physical Fights Scale (HPFS) - past five years and past one year, Hot Sauce Aggression Task (HSAT), NonSuicidal Self-Injury Assessment Tool (NSSIAT), Provocation Manipulation Check (PMC), Taylor Aggression Paradigm (TAP), Voodoo Doll Task (VDT).

\begin{tabular}{llllllll}
\hline & $M$ & $S D$ & Range & $\% 0$ & $\alpha$ & Skew & Kurtosis \\
\hline AMS - Instrument & 2.60 & 1.62 & $1-7$ & - & & 0.95 & 0.12 \\
AMS - Revenge & 2.28 & 1.40 & $1-6.25$ & - & .83 & 0.95 & -0.12 \\
BAQ - Physical & 2.86 & 1.45 & $1-7$ & - & .70 & 0.58 & -0.59 \\
BAQ - Verbal & 3.66 & 1.24 & $1-6.67$ & - & .59 & 0.23 & -0.45 \\
HPFS - Past Five & 0.71 & 1.29 & $0-7$ & 66.70 & - & 2.24 & 5.46 \\
HPFS - Past Year & 0.17 & 0.53 & $0-4$ & 88.10 & - & 3.93 & 18.98 \\
HSAT & 3.82 & 3.90 & $0-20$ & 10.70 & - & 1.67 & 2.90 \\
NSSIAT & 1.21 & 0.86 & $1-7.67$ & - & .97 & 5.47 & 32.77 \\
PMC & 2.47 & 1.93 & $1-7$ & - & .96 & 1.24 & 0.25 \\
TAP & 5.10 & 1.99 & $0-10$ & 1.70 & .98 & -0.36 & 0.48 \\
VDT & 7.41 & 12.04 & $0-51$ & 36.20 & - & 2.17 & 4.37 \\
\hline
\end{tabular}


Group comparisons. Participants who were assigned to the negative feedback condition $(N=87)$ reported greater levels of provocation due to their essay feedback than participants that received positive feedback $(N=90), t(175)=9.75, p<.001, d=$ $1.47[1.20,1.80]$, and had higher TAP scores, $t(175)=3.56, p<.001, d=0.54[0.24$, 0.83; Figure 1]. Males (coded as 1) did not have higher TAP scores than females (coded as 0$), t(175)=-0.93, p=.356, d=-0.14[-0.44,0.16]$. Supplemental Table 2 summarizes gender comparisons in TAP scores within each provocation condition and on self-reported aggression variables.

Figure 1. Violin plots depicting the distributions of TAP scores by essay feedback condition, from Study 1.

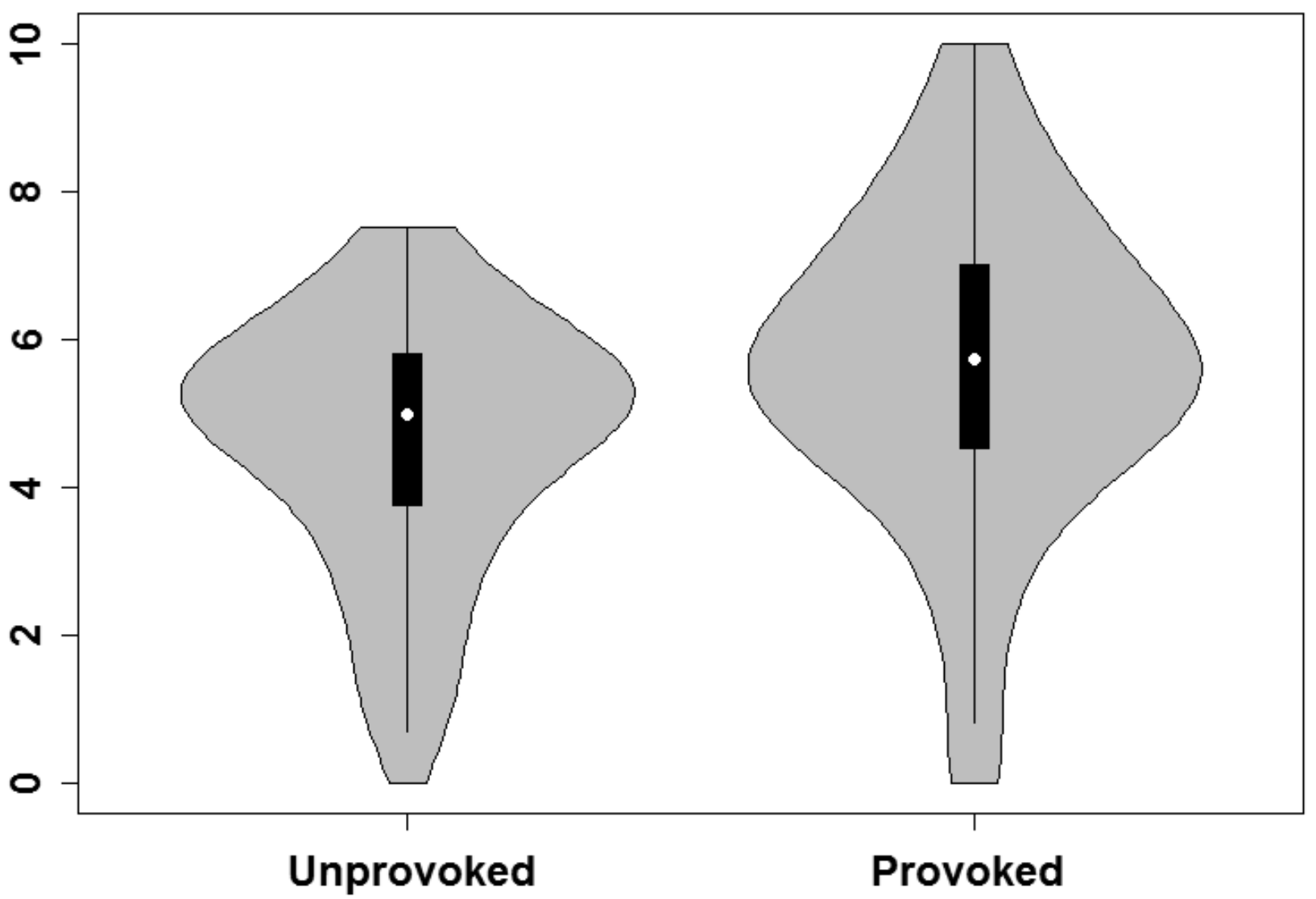


Convergent validity. TAP scores were positively associated with the amount of hot sauce participants gave to their partner, $r(175)=.18[.03, .32], p=.016$ (Figure 2), and with the number of voodoo doll pins (Figure 2, Table 2). TAP scores were unassociated with trait physical aggression, $r(175)=.11[-.04, .25], p=.139$, and positively associated with revengeful aggression motivations, $r(175)=.41[.28, .56], p<$ .001 .

Figure 2. Scatterplots depicting Study 1's positive associations between mean TAP scores and voodoo doll pin counts and hot sauce weights, in grams, directed towards participants' essay evaluators (with and without logarithmic transformation).

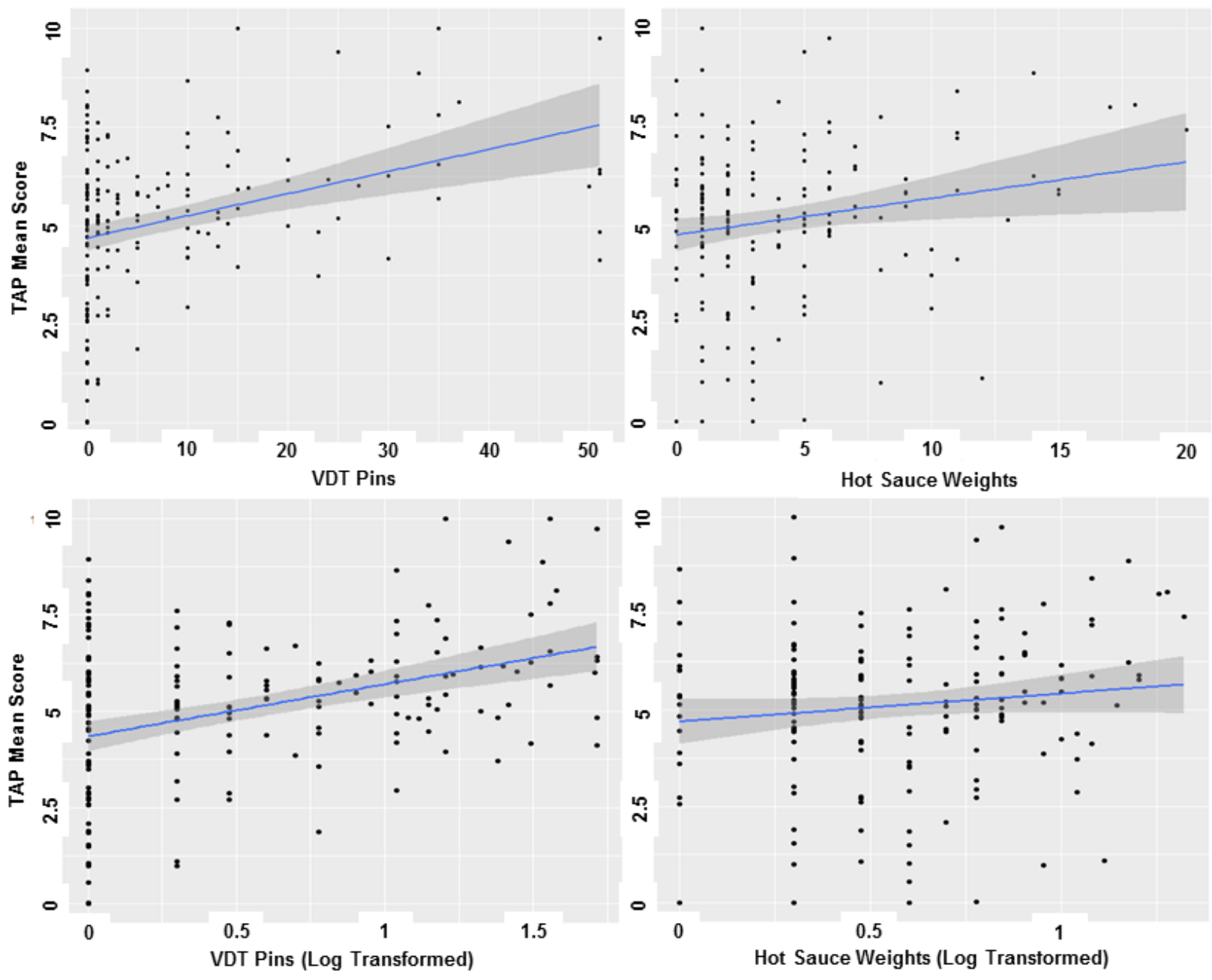


Table 2. Correlations between TAP scores and VDT pin counts from Study 1's generalized linear modeling specifying both Poisson (confirmatory) and negative binomial (exploratory) distributions.

\begin{tabular}{llllll}
\hline \multicolumn{2}{l}{ Poisson Regression } & \multicolumn{5}{l}{ Negative Binomial } \\
\hline$B[95 \% \mathrm{Cl}]$ & $X^{2}$ & $p$ & $B[95 \% \mathrm{Cl}]$ & $X^{2}$ & $p$ \\
\hline $0.30[0.27,0.33]$ & 409.32 & $<.001$ & $0.38[0.28,0.48]$ & 55.46 & $<.001$
\end{tabular}

External \& discriminant validity. Offering mixed evidence of the TAP's external validity, TAP scores were unassociated with the number of physical fights participants had been in over the past 5 years, yet were positively associated with those over the past year (Table 3). TAP scores were unassociated with trait verbal aggression, $r(175)=$ $.10[-.05, .24], p=.204$, yet unexpectedly were positively associated with current selfharm tendencies, $r(175)=.16[.01, .30], p=.032$.

Table 3. Correlations between TAP scores and past physical fight frequencies from Study 1's generalized linear modeling specifying both Poisson (confirmatory) and negative binomial (exploratory) distributions.

\begin{tabular}{lllllll}
\hline Predictor & \multicolumn{2}{l}{ Poisson Regression } & \multicolumn{5}{l}{ Negative Binomial } \\
\hline & $B[95 \% \mathrm{Cl}]$ & $X^{2}$ & $p$ & $B[95 \% \mathrm{Cl}]$ & $X^{2}$ & $p$ \\
\cline { 2 - 7 } Past 5 Years & $-0.00[-0.09,0.08]$ & 0.01 & .934 & $-0.00[-0.12,0.11]$ & 0.00 & .951 \\
Past Year & $0.23[0.04,0.42]$ & 5.66 & .017 & $0.23[0.02,0.44]$ & 4.56 & .033
\end{tabular}

Internal consistency. The 50 individual measurements of the TAP $(25$ trials $\times 2$ responses per trial) exhibited excellent internal consistency (Table 1). A principal components analysis (PCA), with direct oblimin rotation (which allows the extracted components to correlate with one another), was conducted on all 50 TAP settings. The 
data exhibited a single-component structure, as evidenced by a large initial component, which explained almost half of the variance in TAP responses (Figure 3; Supplemental Table 3).

Figure 3. Scree plot of eigenvalues depicting a single component solution for all 50 TAP responses from Study 1.

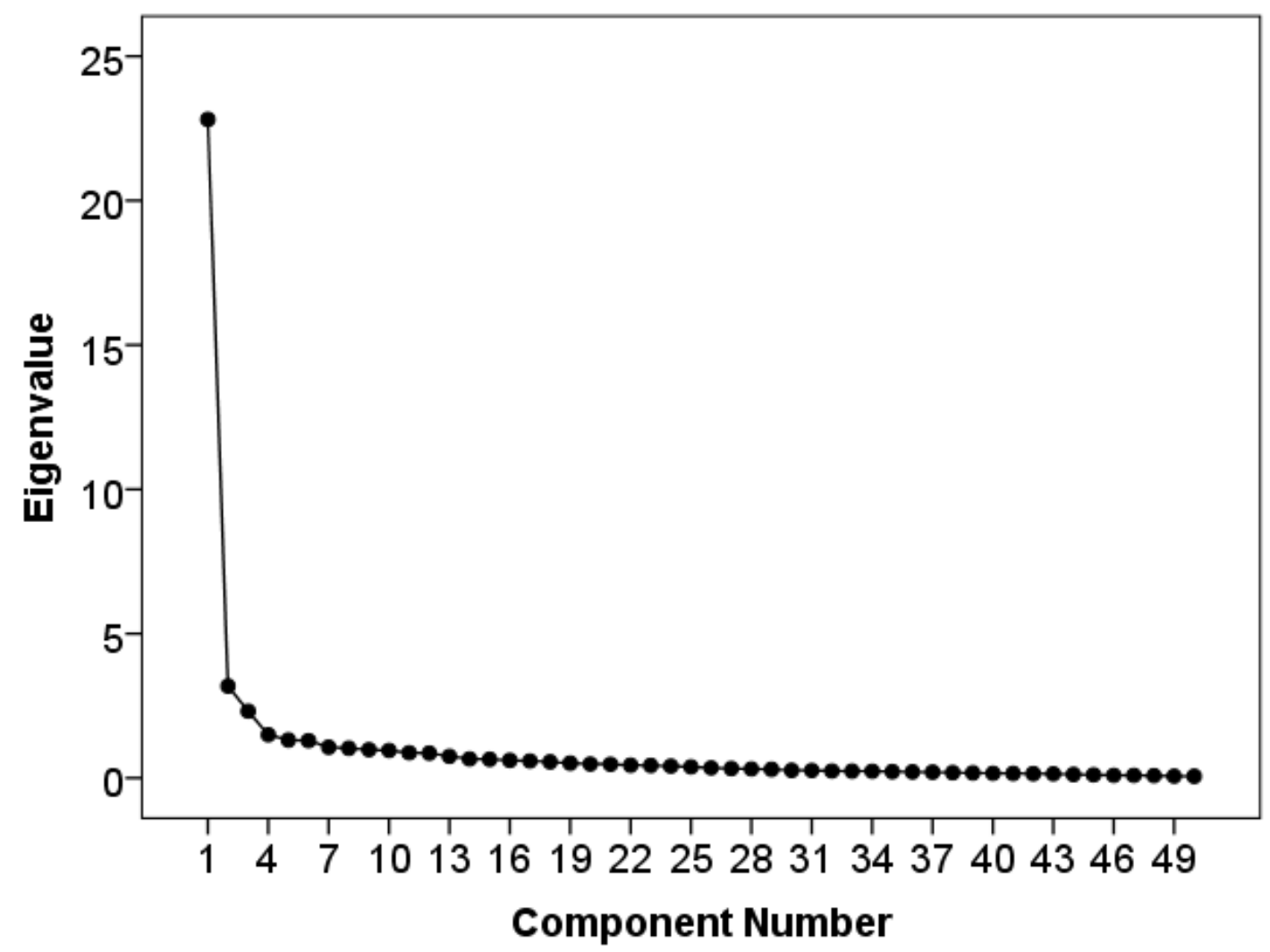

\section{Exploratory Analyses}

Specificity of hot sauce measure to aggression. To assess whether TAP scores were associated with greater hot sauce allocations overall, we correlated TAP scores with participants hot sauce allocations to themselves. Participants' TAP scores were associated with greater hot sauce allocations towards themselves, $r(175)=.31$ $[.17, .44], p<.001$. 
Transformation of aggression variables. Applying logarithmic transformations (after adding 1 to all values to allow for the transformation of 0 values) reduced the skewness and kurtosis of both the voodoo doll pin count and hot sauce weights (Table 4). Transformed voodoo doll pin counts remained significantly associated with TAP scores, $r(175)=.38[.25, .50], p<.001$, whereas transformed hot sauce weights did not, $r(175)=.12[-.03, .26], p=.112$.

Table 4. Study 1 distribution statistics for the hot sauce weights and voodoo doll pins counts after logarithmic transformation. Parenthesized values represent distribution statistics prior to transformation. Normality was assessed via the Shapiro-Wilk Normality Test, $d f=177$, statistical significance indicates non-normality. ${ }^{*} p<.001$

\begin{tabular}{llll}
\hline & Skew & Kurtosis & Non-Normality \\
\hline Hot Sauce Weights & $0.13(1.67)$ & $-0.65(2.90)$ & $0.96^{*}\left(0.82^{*}\right)$ \\
Voodoo Doll Pins & $0.55(2.17)$ & $-1.04(4.37)$ & $0.86^{*}\left(0.66^{*}\right)$ \\
\hline
\end{tabular}

\section{Study 2}

Study 2 was conducted in order to counterbalance the order of the aggression measures and to include additional construct validity measures. The preregistration plan for this study is available online (https://osf.io/ca237/register/565fb3678c5e4a66b5582f67), as are corresponding data, analysis code, and materials (https://osf.io/a2wft/files/).

\section{Methods}

\section{Participants}

In an attempt to recruit at least 160 participants who did not meet exclusionary criteria, we recruited 193 undergraduates from an introductory psychology subject pool, 
ceasing recruitment at the end of the academic semester in which the study took place.

Of these 193 participants, 25 were missing data necessary for our confirmatory analyses ${ }^{1}$ and 4 others expressed 'total disbelief' on our post-debriefing questionnaire (3 were also missing data). Final participants were therefore 167 undergraduates (67.1\% female, $32.9 \%$ male; Age: $M=19.16, S D=2.38$, range: $18-40)$. Participants' racial composition was 44.3\% White, 19.8\% African-American/Black, 19.2\% AsianAmerican, $15.6 \%$ Other, $0.6 \%$ Native American, and $0.6 \%$ declined to respond. The sample was $12.9 \%$ Hispanic and $87.1 \%$ Non-Hispanic. Participants were compensated with course credit.

\section{Measures}

Five Factor Narcissism Inventory. The 60-item FFNI was developed to decomposes narcissistic traits into 15 subfacets that form the broader narcissism dimensions (Glover, Miller, Lynam, Crego, \& Widiger, 2012; Miller et al., 2016). Critical to this study was the antagonism facet, which is constructed by aggregating the arrogance, distrust, entitlement, exploitativeness, lack of empathy, manipulativeness, reactive anger, and thrill-seeking facets.

NEO - Five Factor Inventory. The 60-item NEO-FFI quantifies individuals' levels of agreeableness, conscientiousness, extraversion, neuroticism, and openness to experience (12 items per factor; Costa \& MacCrae, 1992).

Self-Report Psychopathy Scale - III. The 64-item SRP quantifies individuals' psychopathic traits (Paulhus, Neumann, \& Hare, 2009).

${ }^{1}$ Excepting missing data from the Self-Report Psychopathy Scale, Five Factor Narcissism Inventory, and Short Dark Triad 3 questionnaire, due to an excessive amount of missing data. 
Short Dark Triad 3. The 27-item SD3 is a brief measure of the "dark triad" (i.e., Machiavellianism, narcissism, and psychopathy; Jones \& Paulhus, 2014).

\section{Procedure}

The informed consent and provocation manipulation procedures were identical to Study 1. Based on a pre-randomized list that experimenters viewed, participants completed four aggression tasks in six different counterbalancing orders (determined randomly) and each participant was randomly assigned to one of these task orders (Table 5).

Table 5. Counterbalancing orders for the aggression tasks: Buss-Perry Aggression Questionnaire (BPAQ), Hot Sauce Aggression Task (HSAT), Taylor Aggression Paradigm (TAP), Voodoo Doll Task (VDT).

\begin{tabular}{lllllll}
\hline Order & I & II & III & IV & V & VI \\
\hline First & HSAT & HSAT & BPAQ & TAP & TAP & VDT \\
Second & BPAQ & BPAQ & VDT & VDT & BPAQ & BPAQ \\
Third & VDT & TAP & TAP & HSAT & VDT & TAP \\
Fourth & TAP & VDT & HSAT & BPAQ & HSAT & HSAT \\
\hline \multicolumn{5}{c}{ Participants then completed a battery of personality questionnaires and identical }
\end{tabular}
debriefing and suspicion probe procedures to Study 1 .

\section{Results}

\section{Deviations from Preregistration Plan}

The deviations from the preregistration plan that occurred in Study 1 were likewise present in Study 2 due to identical considerations.

\section{Descriptive Statistics}


Descriptive statistics and internal consistency estimates are provided in Table 6. Considerable amounts of data were missing from the personality questionnaires because of time constraints (Table 6). The Aggression Motives Scale was incorrectly administered and the Crime and Analogous Behavior scale had 132 missing datapoints, so both of these measures were excluded from analysis. Zero-order correlations between all study variables are included in Supplemental Table 4.

Table 6. Descriptive statistics for Study 2 confirmatory variables: Buss-Perry Aggression Questionnaire (BPAQ), History of Physical Fights Scale (HPFS) - past five years and ever, Hot Sauce Aggression Task (HSAT), Non-Suicidal Self-Injury Assessment Tool (NSSIAT), Provocation Manipulation Check (PMC), Taylor Aggression Paradigm (TAP), Voodoo Doll Task (VDT).

\begin{tabular}{lllllllll}
\hline & $M$ & $S D$ & Range & $\% 0$ & $\alpha$ & Skew & Kurtosis & N Missing \\
& & & & & & & & \\
\hline BPAQ - Physical & 2.57 & 0.96 & $1.00-5.22$ & - & .79 & 0.52 & -0.43 & 0 \\
BPAQ - Verbal & 3.56 & 1.15 & $1.00-6.60$ & - & .78 & 0.19 & -0.54 & 0 \\
FFNI - Antagonism & 2.24 & 0.67 & $1.09-3.94$ & - & .94 & -0.48 & -0.10 & 94 \\
HPFS - Ever & 2.93 & 10.36 & $0.00-100$ & 47.9 & - & 7.12 & 57.49 & 0 \\
HPFS - Past Five & 1.14 & 4.11 & $0.00-40$ & 68.9 & - & 7.30 & 60.79 & 0 \\
HSAT & 3.73 & 3.59 & $0.00-20$ & 7.2 & - & 1.96 & 4.60 & 0 \\
NEO - Agreeable & 3.63 & 0.45 & $2.50-4.75$ & - & .69 & 0.00 & -0.26 & 0 \\
NSSIAT & 1.07 & 0.29 & $1.00-3.17$ & - & .91 & 5.05 & 28.33 & 0 \\
PMC & 3.00 & 1.46 & $1.00-6.67$ & - & .88 & 0.42 & -0.71 & 9 \\
SD3 - Mach & 2.78 & 0.76 & $1.00-4.89$ & - & .83 & 0.23 & 0.24 & 85
\end{tabular}




$\begin{array}{lllllllll}\text { SRP } & 2.14 & 0.43 & 1.31-3.47 & - & .90 & 0.46 & 0.17 & 62 \\ \text { TAP } & 4.85 & 2.19 & 0-10 & 1.2 & .98 & 0.07 & 0.02 & 0 \\ \text { VDT } & 4.44 & 9.27 & 0-51 & 45.5 & - & 3.43 & 12.86 & 0\end{array}$

Confirmatory Tests

Group comparisons. Participants who were assigned to the negative feedback condition $(N=84)$ reported greater levels of provocation due to their essay feedback than participants in the positive feedback condition $(N=83), t(156)=6.80, p<.001, d=$ $1.09[0.79,1.43]$, and had higher TAP scores, $t(165)=2.10, p=.037, d=0.33[0.02$, 0.63; Figure 4]. Again, males (coded as 1) did not have higher TAP scores than females (coded as 0 ), $t(165)=-0.09, p=.931, d=-0.01[-0.32,0.29]$. Supplemental Table 5 summarizes gender comparisons in TAP scores within each provocation condition and on self-reported aggression variables.

Figure 4. Violin plots depicting the distributions of TAP scores by essay feedback condition, from Study 2. 


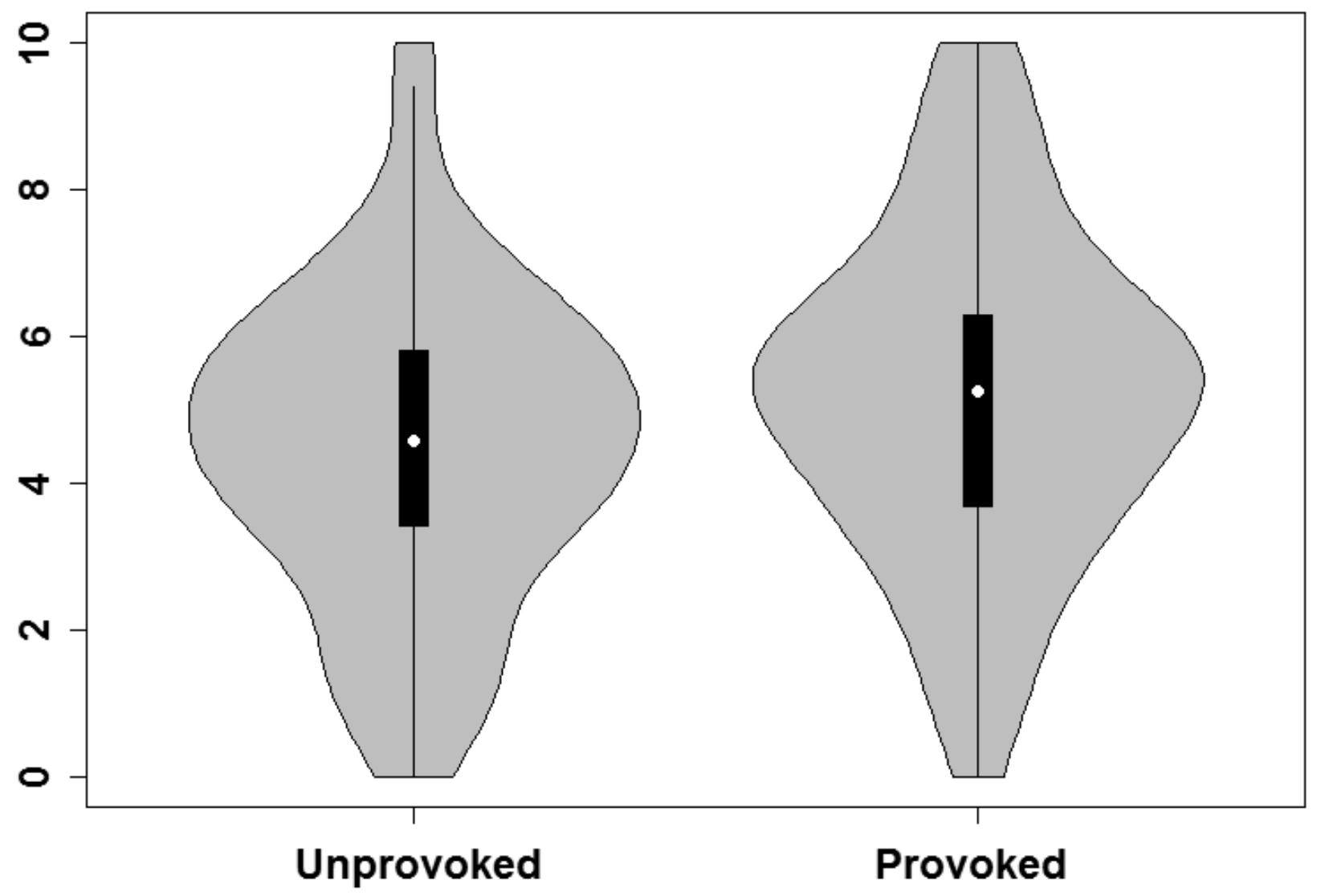

Convergent validity. TAP scores were positively associated with the amount of hot sauce participants gave to their partner in the taste test, $r(165)=.29[.15, .42], p<$ .001 (Figure 5), and with the number of pins stuck in the voodoo doll that represented their partner (Figure 5, Table 7).

Figure 5. Scatterplots depicting Study 2's positive associations between mean TAP scores and voodoo doll pin counts and hot sauce weights, in grams, directed towards participants' essay evaluators (with and without logarithmic transformation). 


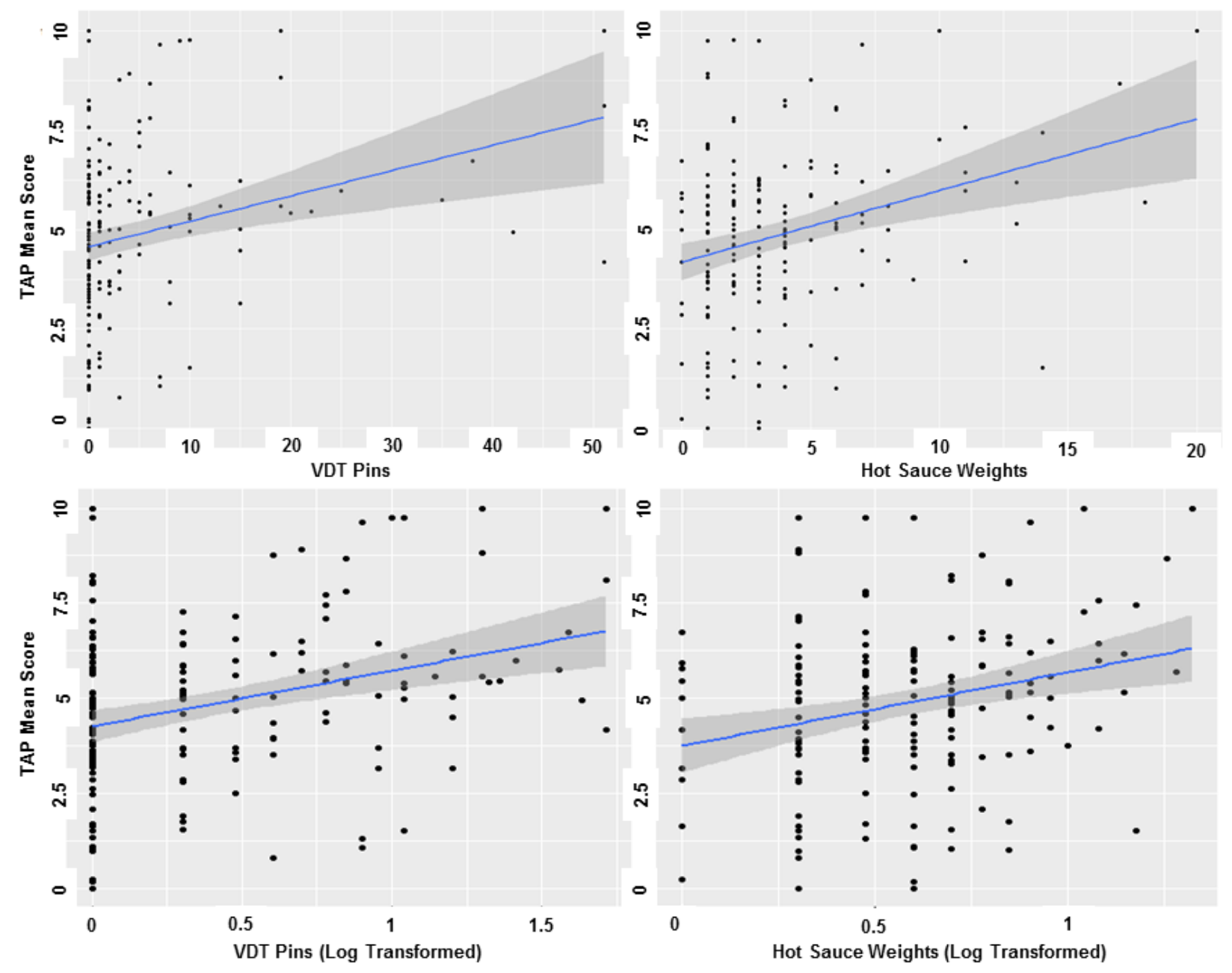

Table 7. Correlations between TAP scores and VDT pin counts from Study 2's generalized linear modeling specifying both Poisson (confirmatory) and negative binomial (exploratory) distributions modeling.

\begin{tabular}{llllll}
\hline \multicolumn{2}{l}{ Poisson Regression } & \multicolumn{5}{l}{ Negative Binomial } \\
\hline$B[95 \% \mathrm{Cl}]$ & $X^{2}$ & $p$ & $B[95 \% \mathrm{Cl}]$ & $X^{2}$ & $p$ \\
\hline $0.25[0.22,0.29]$ & 232.13 & $<.001$ & $0.27[0.19,0.36]$ & 39.49 & $<.001$
\end{tabular}

Personality traits. As in Study 1, TAP scores were unassociated with trait physical aggression, $r(165)=.15[-.00, .30], p=.055$. Further, TAP scores were negatively associated with trait agreeableness, $r(165)=-.19[-.33,-.04], p=.015$, 
positively associated with the antagonistic facet of trait narcissism, $r(71)=.40[.19, .58]$, $p<.001$, and unassociated with Machiavellianism, $r(80)=.16[-.06, .36], p=.143$, or psychopathy, $r(103)=.08[-.11, .27], p=.393$.

External and discriminant validity. TAP scores were unassociated with the number of physical fights participants had been in over the past 5 years, yet TAP scores were positively associated with fights that participants had ever been in (Table 8). TAP scores were unassociated with trait verbal aggression, $r(165)=.12[-.03, .27], p=.134$, and current self-harm tendencies ${ }^{2}, r(165)=.03[-.12, .18], p=.691$.

Table 8. Correlations between TAP scores and past physical fight frequencies from Study 2's generalized linear modeling specifying both Poisson (confirmatory) and negative binomial (exploratory) distributions.

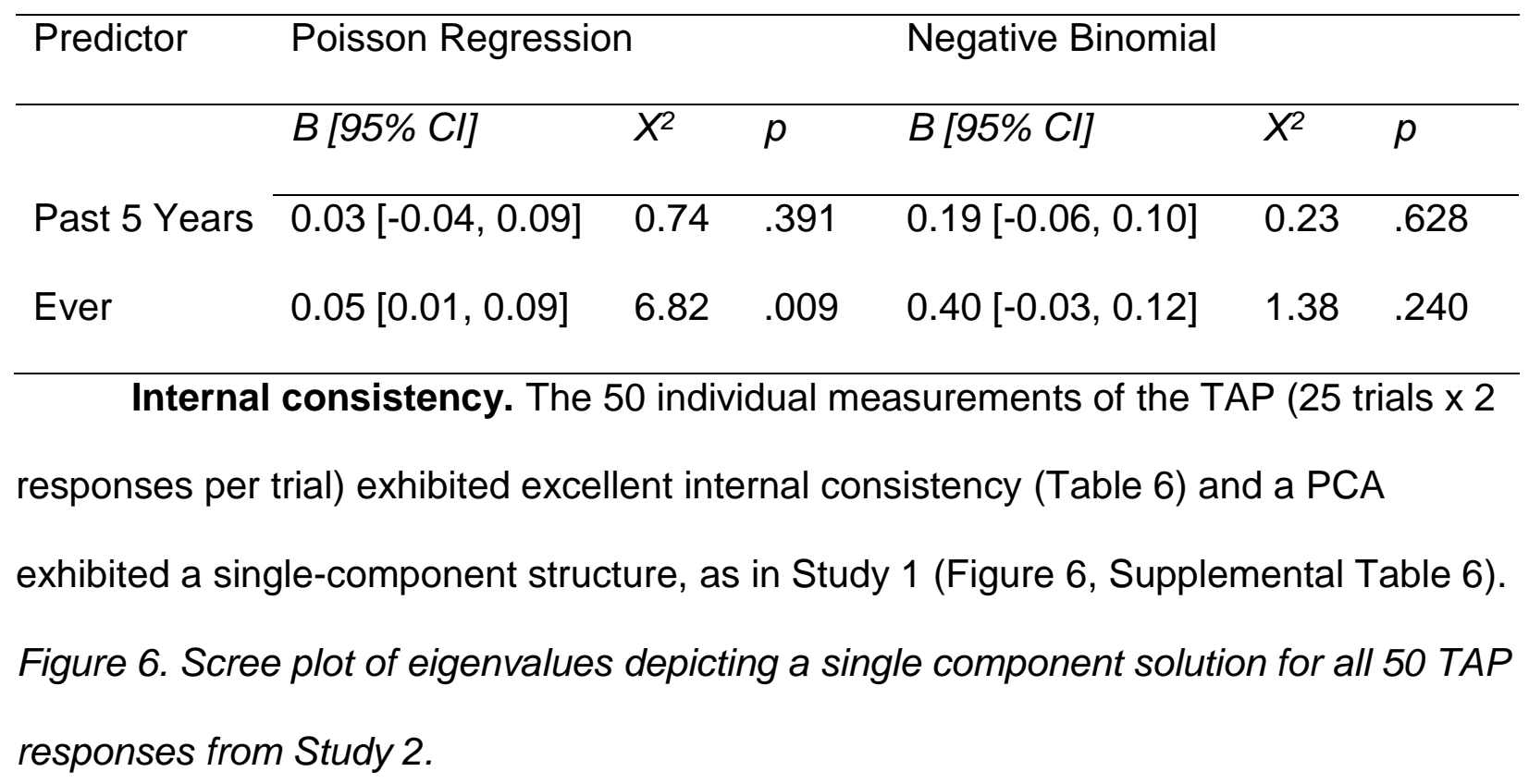

\footnotetext{
${ }^{2}$ NSSIAT responses in Study 2 were provided along a 1-5 response scale, instead of the 1-9 response scale used in Study 1.
} 


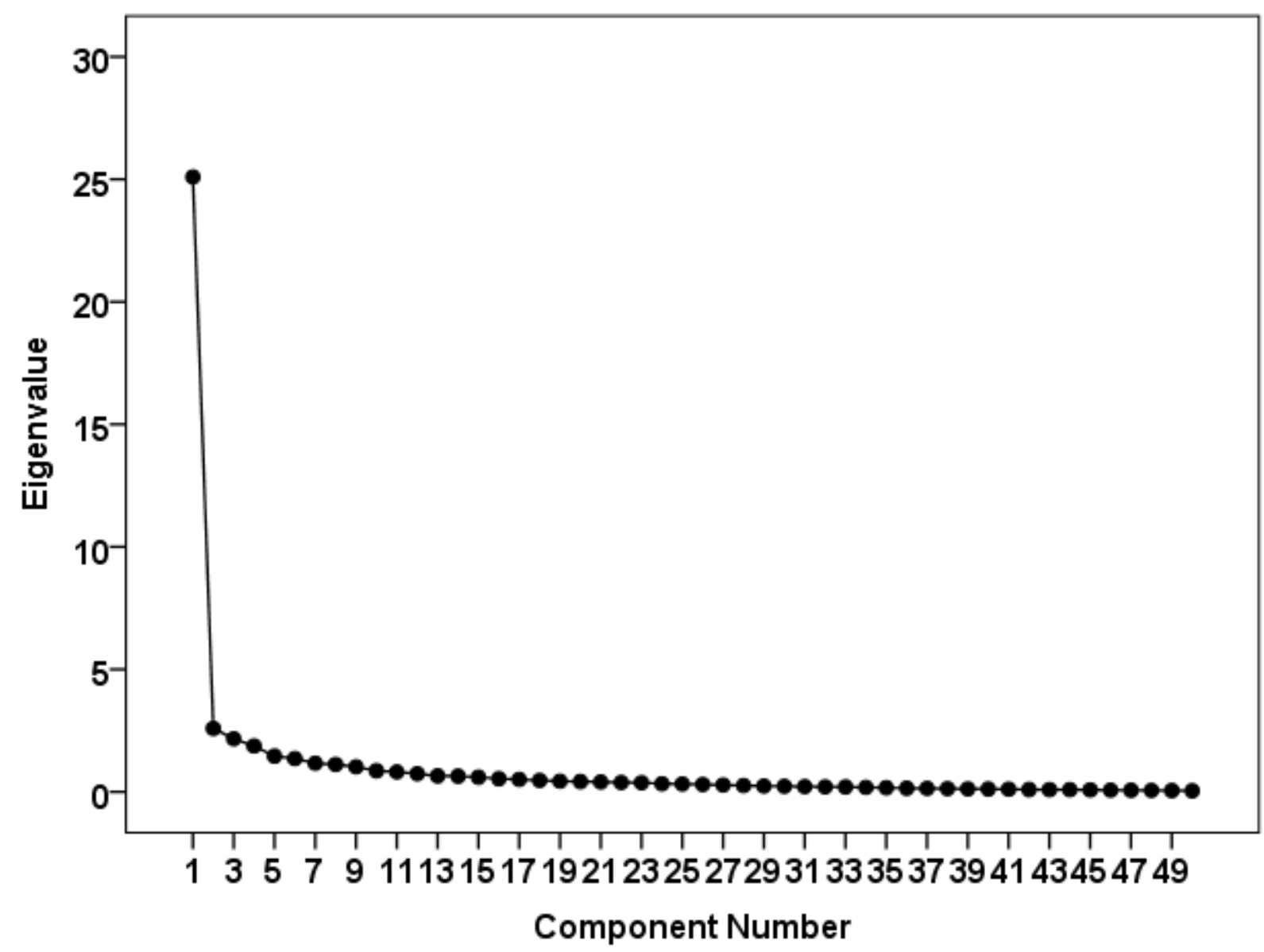

\section{Exploratory Analyses}

Specificity of hot sauce measure to aggression. Unlike Study 1, participants' TAP scores were unassociated with greater hot sauce allocations towards themselves, $r(165)=.14[-.01, .29], p=.075$

Transformation of aggression variables. Applying logarithmic transformations reduced the skewness and kurtosis of both the voodoo doll pin count and hot sauce weights (Table 9). Transformed voodoo doll pin counts remained significantly associated with TAP scores, $r(165)=.32[.18, .45], p<.001$, as did transformed hot sauce weights, $r(165)=.26[.11, .40], p=.001$. 
Table 9. Study 2 distribution statistics for the hot sauce weights and voodoo doll pins counts after logarithmic transformation. Parenthesized values represent distribution statistics prior to transformation. Normality was assessed via the Shapiro-Wilk Normality Test, $d f=167 .^{*} p<.001$

\begin{tabular}{llll}
\hline & Skew & Kurtosis & Non-Normality \\
\hline Hot Sauce Weights & $0.12(1.96)$ & $-0.25(-0.84)$ & $0.97^{\star}\left(0.80^{\star}\right)$ \\
Voodoo Doll Pins & $0.98(3.43)$ & $-0.18(12.86)$ & $0.82^{\star}\left(0.52^{*}\right)$ \\
\hline
\end{tabular}

\section{Order Effects}

A one-way ANOVA revealed that TAP scores were unaffected by the order of aggression tasks, $F(5,160)=1.30, p=.267$.

\section{Internal Meta-Analysis}

An internal, fixed-effects meta-analysis across both studies' zero-order correlations between TAP scores and each key variable measured in both studies was performed, weighted by sample size (Goh, Hall, \& Rosenthal, 2016). Each correlation coefficient was Fisherian Z-transformed for analysis and then converted back into the original correlation coefficient for presentation's sake (Table 10).

Table 10. Internal meta-analysis on both studies' key variables: Buss-Perry Aggression Questionnaire (BPAQ), Hot Sauce Aggression Task (HSAT), Non-Suicidal Self-Injury Assessment Tool (NSSIAT), Voodoo Doll Task (VDT). Correlations were weighted by sample size, $Z$ tests represent significance tests of each meta-analytic effect, and the $X^{2}$ tests represent tests for heterogeneity between studies.

$$
r[95 \% C l] \quad Z(p) \quad X^{2}(p)
$$




$\begin{array}{llll}\text { BPAQ - Physical } & .13[.03, .23] & 2.41(.016) & 0.14(.708) \\ \text { BPAQ - Verbal } & .11[.01, .21] & 2.04(.041) & 0.03(.862) \\ \text { HSAT (Untransformed) } & .24[.14, .34] & 4.34(<.001) & 1.15(.284) \\ \text { HSAT (LogTransformed) } & .19[.09, .29] & 3.50(<.001) & 1.79(.181) \\ \text { Male } & -.04[-.15, .07] & -0.75(.453) & 0.31(.578) \\ \text { NSSIAT } & .10[-.01, .20] & 1.78(.075) & 1.46(.227) \\ \text { Provocation } & .22[.12, .32] & 3.91(<.001) & 0.93(.335) \\ \text { VDT (Untransformed) } & .32[.22, .41] & 5.67(<.001) & 0.51(.475) \\ \text { VDT (LogTransformed) } & .37[.28, .46] & 6.50(<.001) & 0.40(.527)\end{array}$

\section{Integrative Data Analyses}

We also performed integrative data analyses by combining both study's datasets, standardizing each predictor within-study, and using multilevel modeling in SAS v.9.4, modeling the following sources of variance: participant (level 1), study (level 2; Curran \& Hussong, 2009). We tested linear effects of each predictor on TAP scores, as well separate, exploratory models that included a squared version of the predictor to examine potential quadratic effects. These analyses largely replicated the results of the internal meta-analyses and revealed mixed incremental support for quadratic effects (Table 11).

Table 11. Integrative data analysis on both studies' key variables: Buss-Perry Aggression Questionnaire (BPAQ), Hot Sauce Aggression Task (HSAT), Non-Suicidal Self-Injury Assessment Tool (NSSIAT), Voodoo Doll Task (VDT).

Linear Quadratic




\begin{tabular}{lllllll}
\hline & $\beta$ & $t$ & $p$ & $\beta$ & $t$ & $p$ \\
\hline Linear Effect Only & & & & & & \\
& & & & & & \\
\hline BPAQ - Physical & 0.35 & 3.12 & .002 & - & - & - \\
BPAQ - Verbal & 0.22 & 2.01 & .047 & - & - & - \\
HSAT (Untransformed) & 0.50 & 4.56 & $<.001$ & - & - & - \\
HSAT (LogTransformed) & 0.40 & 3.63 & $<.001$ & - & - & - \\
NSSIAT & 0.20 & 1.77 & .080 & - & - & - \\
VDT (Untransformed) & 0.63 & 5.91 & $<.001$ & - & - & - \\
VDT (LogTransformed) & 0.72 & 6.86 & $<.001$ & - & - & - \\
\hline Linear + Quadratic Effects & & & & & & \\
\hline BPAQ - Physical & 0.32 & 2.59 & .011 & 0.07 & 0.61 & .541 \\
BPAQ - Verbal & 0.17 & 1.51 & .134 & 0.19 & 1.69 & .095 \\
HSAT (Untransformed) & 0.34 & 2.01 & .047 & 0.21 & 1.25 & .215 \\
HSAT (LogTransformed) & 0.37 & 3.37 & .001 & 0.31 & 2.82 & .006 \\
NSSIAT & 0.11 & 0.36 & .717 & 0.10 & 0.35 & .730 \\
VDT (Untransformed) & 1.19 & 5.34 & $<.001$ & -0.64 & -2.84 & .005 \\
VDT (LogTransformed) & 0.70 & 5.17 & $<.001$ & 0.04 & 0.27 & .791 \\
\hline & & & & & & \\
\hline
\end{tabular}

Quadratic associations between TAP scores and transformed hot sauce allocations and raw VDT pin counts were observed (Supplemental Figure 1). These paradoxical, at-odds findings may reflect the extent to which extreme levels of aggression exhibited on the TAP may not accurately capture aggressive behavior, given that they correlate with both heavier hot sauce weights and fewer voodoo doll pins. 
Confirmatory factor analyses (CFA) were fit with maximum likelihood estimation in AMOS v.24.0.0 to identify the underlying data structure and relationships among the aggression variables in this study. Variance estimates for each latent factor were fixed to 1 .

\section{Measurement Models}

TAP data from both studies were combined to assess the fit of several measurement CFAs: a one-factor approach in which all 50 TAP measurements load onto a single latent 'aggression' construct, a two-factor approach in which the volume and duration settings from the first trial loaded onto a separate 'trial 1' latent factor and the remaining trials loaded onto a second ' 24 trials' factor, a three-factor model that also modeled trial 2 settings as their own latent construct, and a final two-factor approach in which all volume settings load onto a 'volume' latent construct and all duration settings load onto a 'duration' latent construct (error terms were allowed to covary within each trial). Insufficient model fit was observed for all models (Table 12), though the final model exhibited the best fit (volume and duration factors were highly-correlated, $r=.93$ ). Across all models, each TAP variable's loading onto each given latent construction was positive and statistically-significant.

Table 12. Model fit statistics for various models of TAP data from both studies. CFI = Comparative Fit Index. RMSEA = Root Mean Square Error of Approximation, $T L I=$ Tucker-Lewis Index. Values in brackets represent $90 \%$ confidence interval. ${ }^{* * *} p<.001$

\begin{tabular}{llllll}
\hline & $X^{2}$ & CFI & RMSEA & TLI & $X^{2}$ Comparison \\
\hline One-Factor & $4,052.09^{* \star *}$ & .79 & .09 & .77 & n/a
\end{tabular}


$[.08, .09]$

Trial 1 as Separate Factor

$4,052.09^{\star \star \star} \quad .79 \quad .09$

$.77 \quad 0$

$[.08, .09]$

Trials 1 and 2 as Separate Factors

$4,016.98^{\star \star *} \quad .79 \quad .09$

$.7735 .11^{\star \star *}$

$[.08, .09]$

Volume/Duration as Separate Factors

$3,569.82^{\star * *} \quad .83 \quad .08$

$.81482 .27^{\star \star *}$

$[.08, .08]$

\section{Taxonomic Approach to Aggression Measurement}

To understand the underlying nomological network underlying the aggression measures employed across both studies (TAP scores, log-transformed HSAT weights, log-transformed VDT pin counts, Physical and Verbal subscales of the BPAQ), we conducted two CFAs. CFAs identified that a general aggression factor did not exhibit adequate fit to the data (Figure 7, Table 13). However, a two-factor model that separately modeled self-report and behavioral measures exhibited better, though still inadequate model fit, $X^{2}(1)=37.69, p<.001$.

Table 13. Model fit statistics for one- and two-factor models of aggression data from both studies. $\mathrm{CFI}=$ Comparative Fit Index. RMSEA = Root Mean Square Error of Approximation, $T L I=$ Tucker-Lewis Index. Values in brackets represent 90\% confidence intervals.

\begin{tabular}{llllll}
\hline & $X^{2}(d f), p$ & CFI & GFI & $R M S E A$ & $T L I$ \\
\hline One-Factor & $47.56(5),<.001$ & .67 & .95 & $.16[.12, .20]$ & .34 \\
Two-Factor & $9.87(4), .043$ & .96 & .99 & $.07[.01, .12]$ & .89 \\
\hline
\end{tabular}


Figure 7. Confirmatory factor analyses that estimate one-factor (left panel) and twofactor (right panel) models of all five aggression measures used in both studies. Values above paths represent standardized coefficients and values attached to variables represent residual, unstandardized variances. Dashed paths are non-significant. ${ }^{*} p<$ $.05,{ }^{* *} p<.01,{ }^{* *} p<.001$.
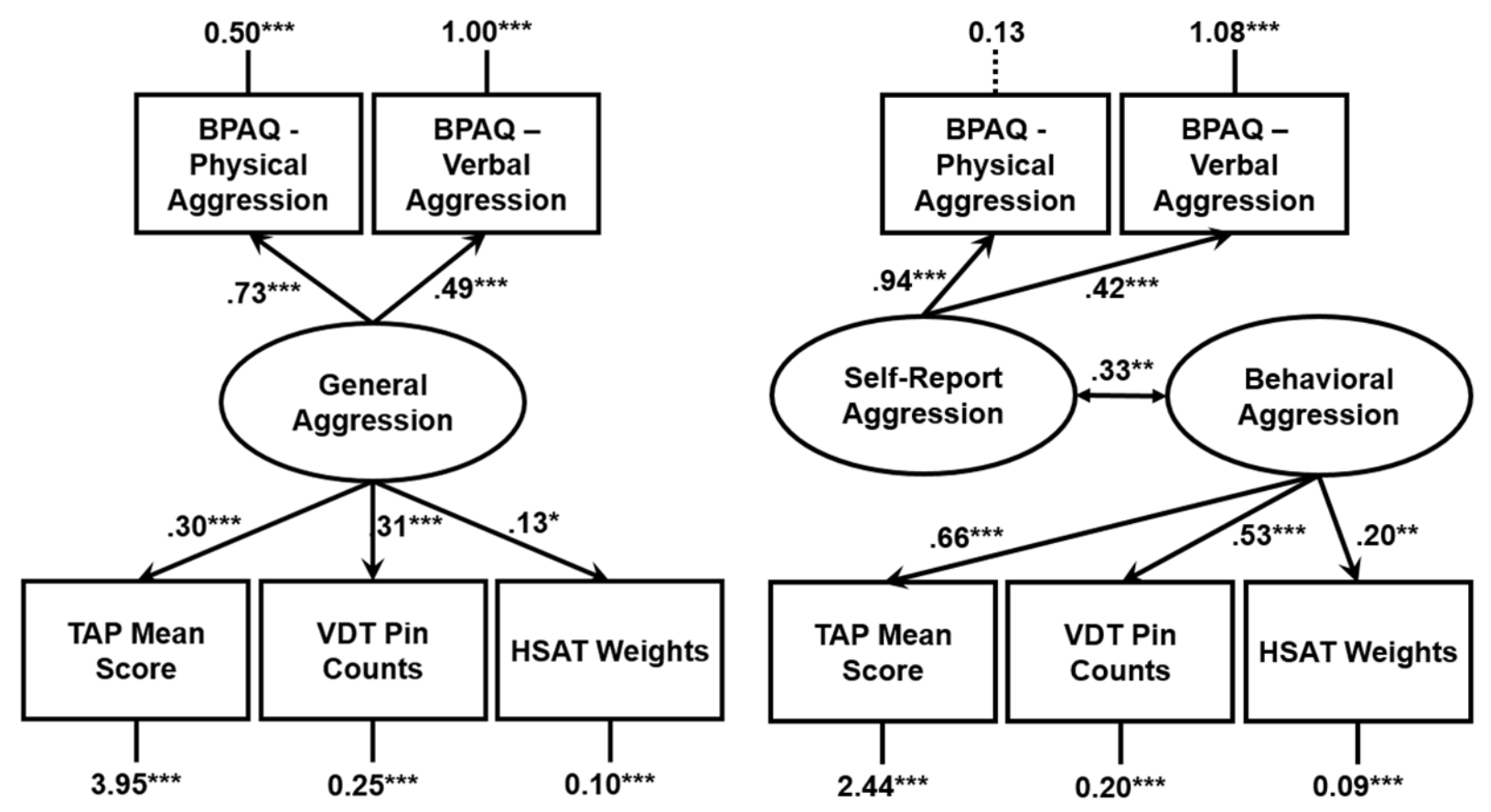

Discussion

The TAP has facilitated substantial developments in our understanding of aggression. However, the ability to implement and analyze the TAP in a nearly unlimited array of ways is both a strength and a weakness. The TAP's flexibility can enable researcher degrees-of-freedom, whether conscious or incidental, to undermine the validity of the task and introduce false positives into the literature (Elson et al., 2014). Using a preregistered version of the TAP and a single scoring approach (i.e., averaging 
across all measurements), we removed the flexibility of the task and tested its subsequent validity in two studies.

Construct and convergent validity. We observed substantial evidence for the construct validity of the aggregate approach to the 25-trial TAP. TAP scores were higher for experimentally provoked participants, as compared to their non-provoked counterparts. Further, the TAP demonstrated convergent validity with the two other laboratory aggression measures: the Hot Sauce Aggression Task (Lieberman et al., 1999) and the Voodoo Doll Aggression Task (DeWall et al., 2013), even after counterbalancing them. This convergence of a triad of laboratory aggression measures suggests that aggression can indeed be operationalized in many ways that tap, that likely tap into a single, underlying construct. In Study 1, though not Study 2, TAP scores were also associated with greater hot sauce allocations from participants to themselves, which may suggest that this task is not purely capturing aggressive behavior. Extending into self-reports, trait physical aggressiveness was also associated with greater TAP scores, suggesting that this task can tap into broader domains of aggressive tendencies.

In Study 2, TAP scores were associated with less agreeableness and greater narcissistic antagonism. These correlations fit with a broader literature demonstrating that antagonistic, disagreeable traits lie at the heart of aggressive behavior (Vize et al., in press). Null effects were observed for psychopathy and Machiavellianism, against predictions, potentially due to the missing data from Study 2 . Additionally, our use of a strong provocation manipulation, coupled with the provocation embedded into the TAP itself, likely constrained the influence of individual difference factors on aggression 
(Saleem, Barlett, Anderson, \& Hawkins, 2017). Attempts at identifying the trait correlates of the TAP would likely benefit from the removal of any situational provocation inductions.

Motivation. TAP scores corresponded to the appropriate motivation, the desire to inflict retributive harm, and not with competitive or instrumental motives to simply win the competition. These findings suggest that TAP scores do not reflect strategic concerns, but instead reflect the motivation to harm one's opponent. Moreover, these results support the validity of the TAP as an effective measure of state-level, immediate aggressive tendencies.

Gender. Males and females did not exhibit differences in their TAP scores. The disproportionate level of females in the sample may have contributed to this null effect. More likely, this lack of a gender difference reflects the complicated nature of gender in aggressive behavior (Archer, 2000).

External validity. On both studies, TAP scores failed to predict frequency of physical fights over the past 5 years, yet was able to do so in shorter (past year) and longer (ever) timeframes. Our sample consisted of undergraduates, who cannot be characterized as a population prone to extreme violence, which may have influenced these results. Their lack of aggressive tendencies likely influenced our results in ways (e.g., restriction-of-range of TAP scores) that can only be estimated by future work with other populations (e.g., violent offenders).

Discriminant validity. Mixed evidence was also observed for the TAP's discriminant validity. In the meta-analysis, TAP scores were modestly associated with trait verbal aggressiveness and marginally associated with state-level self-harm 
tendencies. However, given the frequent association between physical aggression and both of these constructs (e.g., Muehlenkamp \& Gutierrez, 2007; Webster et al., 2014), these associations perhaps should have been expected.

Internal consistency. Principal components analyses and internal consistency estimates suggested that the 50 datapoints of the TAP do, in fact, load onto a central latent construct. As such, our scoring approach of averaging across all 50 datapoints likely accurately reflects the underlying data structure. More research is needed to test whether different trials of the TAP represent quantitatively or qualitatively different measures.

\section{Conclusions}

Flexible psychological measures are a boon to the field. However, this flexibility must be tempered with preregistration of the task's implementation, scoring, and analysis, lest this flexibility undermine sound science. Using two preregistered studies, we provided initial evidence that our approach to the TAP is internally-valid. However, more work is needed to explore the TAP's external and discriminant validity. Assuming that evidence for these other forms of validity are found and that our findings are replicated by future work, the TAP should have a long and healthy life in the aggression researcher's toolkit. 


\section{References}

Anderson, C. A., \& Bushman, B. J. (1997). External validity of "trivial" experiments: The case of laboratory aggression. Review of General Psychology, 1(1), 19-41.

Anderson, C. A., \& Murphy, C. R. (2003). Violent video games and aggressive behavior in young women. Aggressive Behavior, 29(5), 423-429.

Bernstein, S., Richardson, D., \& Hammock, G. (1987). Convergent and discriminant validity of the Taylor and Buss measures of physical aggression. Aggressive Behavior, 13(1), 15-24.

Bond, A., \& Lader, M. (1986). A method to elicit aggressive feelings and behaviour via provocation. Biological Psychology, 22(1), 69-79.

Bushman, B. J. (1995). Moderating role of trait aggressiveness in the effects of violent media on aggression. Journal of Personality and Social Psychology, 69(5), 950960.

Bushman, B. J., \& Baumeister, R. F. (1998). Threatened egotism, narcissism, selfesteem, and direct and displaced aggression: Does self-love or self-hate lead to violence? Journal of Personality and Social Psychology, 75(1), 219-229.

Chester, D. S. \& DeWall, C. N. (2016). The pleasure of revenge: Retaliatory aggression arises from a neural imbalance toward reward. Social Cognitive and Affective Neuroscience, 11(7), 1173-1182.

Chester, D. S. \& DeWall, C. N. (2017). Combating the sting of rejection with the pleasure of revenge: A new look at how emotion shapes aggression. Journal of Personality and Social Psychology, 112(3), 413-430. 
Chester, D. S., Whitt, Z. T., Davis, T. S., \& DeWall, C. N. (2017). The Voodoo Doll SelfInjury Task: A new measure of sub-clinical self-harm tendencies. Journal of Social and Clinical Psychology, 36(7), 554-578.

Costa, P. T., \& MacCrae, R. R. (1992). Revised NEO personality inventory (NEO PI-R) and NEO five-factor inventory (NEO-FFI): Professional manual. Psychological Assessment Resources, Incorporated.

Curran, P. J., \& Hussong, A. M. (2009). Integrative data analysis: the simultaneous analysis of multiple data sets. Psychological Methods, 14(2), 81-100.

Denson, T. F., von Hippel, W., Kemp, R. I., \& Teo, L. S. (2010). Glucose consumption decreases impulsive aggression in response to provocation in aggressive individuals. Journal of Experimental Social Psychology, 46(6), 1023-1028.

Elson, M., Mohseni, M. R., Breuer, J., Scharkow, M., \& Quandt, T. (2014). Press CRTT to measure aggressive behavior: The unstandardized use of the competitive reaction time task in aggression research. Psychological Assessment, 26(2), 419.

Epstein, S., \& Taylor, S. P. (1967). Instigation to aggression as a function of degree of defeat and perceived aggressive intent of the opponent1. Journal of Personality, 35(2), 265-289.

Ferguson, C. J., Smith, S., Miller-Stratton, H., Fritz, S., \& Heinrich, E. (2008). Aggression in the laboratory: Problems with the validity of the modified Taylor Competitive Reaction Time Test as a measure of aggression in media violence studies. Journal of Aggression, Maltreatment \& Trauma, 17(1), 118-132. 
Giancola, P. R., \& Parrott, D. J. (2008). Further evidence for the validity of the Taylor Aggression Paradigm. Aggressive Behavior, 34(2), 214-229.

Giancola, P. R., \& Zeichner, A. (1995). Construct validity of a competitive reaction-time aggression paradigm. Aggressive Behavior, 21(3), 199-204.

Glover, N., Miller, J. D., Lynam, D. R., Crego, C., \& Widiger, T. A. (2012). The five-factor narcissism inventory: A five-factor measure of narcissistic personality traits. Journal of personality assessment, 94(5), 500-512.

Goh, J. X., Hall, J. A., \& Rosenthal, R. (2016). Mini meta-analysis of your own studies: Some arguments on why and a primer on how. Social and Personality Psychology Compass, 10(10), 535-549.

Jones, D. N., \& Paulhus, D. L. (2014). Introducing the short dark triad (SD3) a brief measure of dark personality traits. Assessment, 21(1), 28-41.

Lieberman, J. D., Solomon, S., Greenberg, J., \& McGregor, H. A. (1999). A hot new way to measure aggression: Hot sauce allocation. Aggressive Behavior, 25(5), 331348.

Miller, J. D., \& Lynam, D. R. (2006). Reactive and proactive aggression: Similarities and differences. Personality and Individual Differences, 41(8), 1469-1480.

Miller, J. D., Lynam, D. R., McCain, J. L., Few, L. R., Crego, C., Widiger, T. A., \& Campbell, W. K. (2016). Thinking structurally: A test of the factor structure of the Five-Factor Narcissism Inventory. Journal of Personality Disorders, 30, 1-18.

Muehlenkamp, J. J., \& Gutierrez, P. M. (2007). Risk for suicide attempts among adolescents who engage in non-suicidal self-injury. Archives of Suicide Research, 11(1), 69-82. 
Paulhus, D. L., Neumann, C. S., \& Hare, R. D. (2009). Manual for the self-report psychopathy scale. Toronto: Multi-health systems.

Rozin, P., Millman, L., \& Nemeroff, C. (1986). Operation of the laws of sympathetic magic in disgust and other domains. Journal of Personality and Social Psychology, 50(4), 703-712.

Saleem, M., Barlett, C. P., Anderson, C. A., \& Hawkins, I. (2017). Helping and hurting others: person and situation effects on aggressive and prosocial behavior as assessed by the Tangram task. Aggressive Behavior, 43(2), 133-146.

Shortell, J., Epstein, S., \& Taylor, S. P. (1970). Instigation to aggression as a function of degree of defeat and the capacity for massive retaliation. Journal of Personality, 38(3), 313-328.

Simmons, J. P., Nelson, L. D., \& Simonsohn, U. (2011). False-positive psychology: Undisclosed flexibility in data collection and analysis allows presenting anything as significant. Psychological Science, 22(11), 1359-1366.

Taylor, S. P. (1967). Aggressive behavior and physiological arousal as a function of provocation and the tendency to inhibit aggression. Journal of Personality, 35(2), 297-310.

Tedeschi, J. T., \& Quigley, B. M. (1996). Limitations of laboratory paradigms for studying aggression. Aggression and Violent Behavior, 1(2), 163-177.

Tedeschi, J. T., \& Quigley, B. M. (2000). A further comment on the construct validity of laboratory aggression paradigms: A response to Giancola and Chermack. Aggression and Violent Behavior, 5(2), 127-136. 
Vize, C. E., Collison, K. L., Crowe, M. L., Campbell, W. K., Miller, J. D., \& Lynam, D. R. (in press). Using dominance analysis to decompose narcissism and its relation to aggression and externalizing outcomes. Assessment.

Wagenmakers, E.-J., Wetzels, R., Borsboom, D., van der Maas, H. L. J., \& Kievit, R. A. (2012). An agenda for purely confirmatory research. Perspectives on Psychological Science, 7(6), 632-638.

Webster, G. D., DeWall, C. N., Pond, R. S., Deckman, T., Jonason, P. K., Le, B. M., ... Bator, R. J. (2014). The brief aggression questionnaire: Psychometric and behavioral evidence for an efficient measure of trait aggression. Aggressive Behavior, 40(2), 120-139. 
Supplemental Figure 1. Quadratic associations between mean TAP scores and (A) logtransformed hot sauce weights and (B) un-transformed voodoo doll pin counts, from both studies. The middle line represents the quadratic regression line and the lines on either side demarcate the boundaries of the $95 \%$ confidence interval around this regression line.
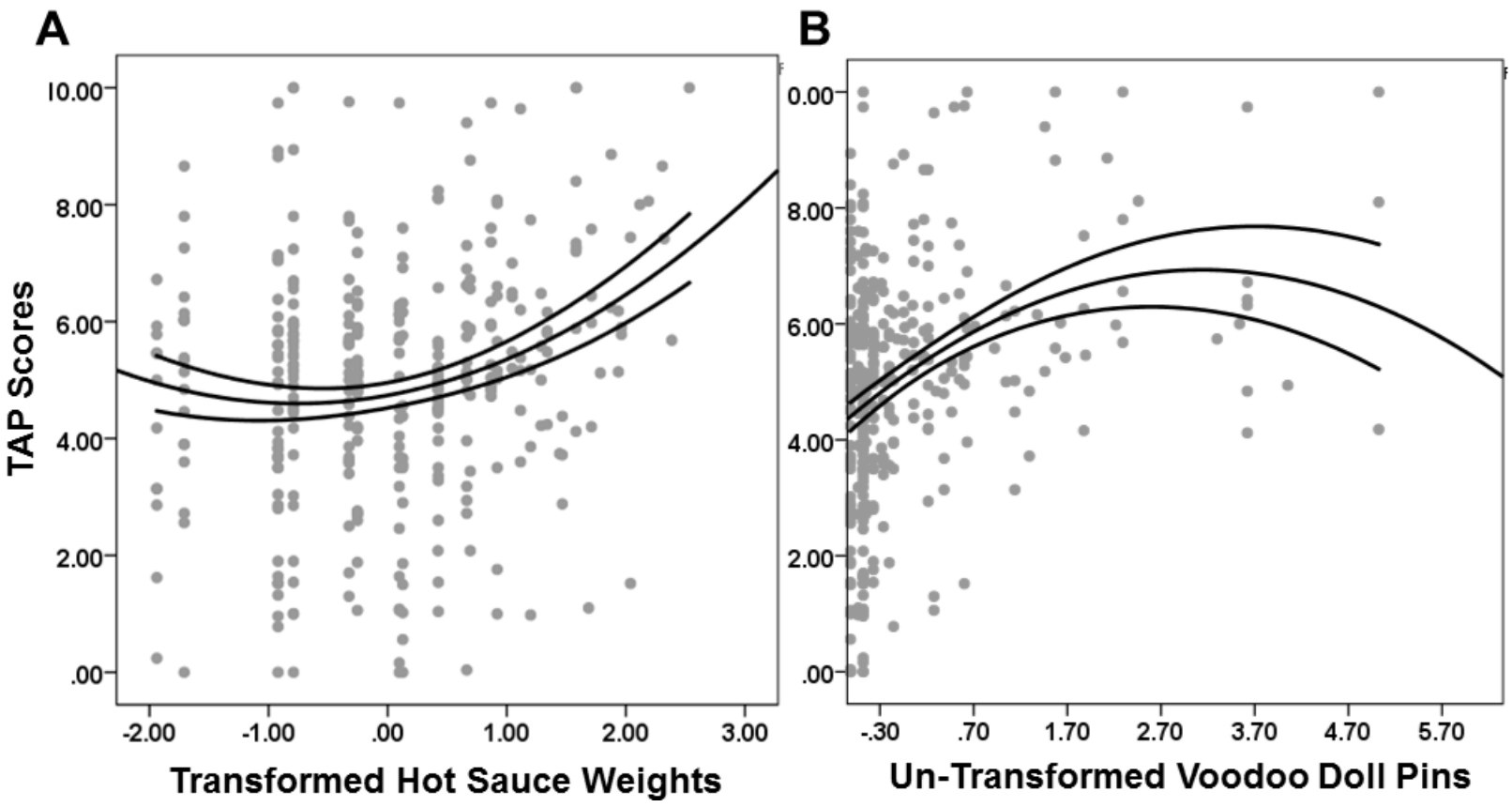
Supplemental Table 1: https://osf.io/a2wft/files/ 
Supplemental Table 2. Gender differences in self-reported aggression variables from Study 1 , as well as gender differences in TAP scores presented separately by provocation condition $($ Male $=1$, Female $=0) . B P A Q=$ Buss-Perry Aggression Questionnaire, HPFS = History of Physical Fights Scale, TAP = Taylor Aggression Paradigm.

\begin{tabular}{lllll}
\hline Variable & $t$ & $d f$ & $p$ & $d[95 \% \mathrm{Cl}]$ \\
\hline BPAQ - Physical Aggression & 4.45 & 175 & $<.001$ & $0.67[0.36,0.95]$ \\
BPAQ - Verbal Aggression & 1.80 & 175 & .073 & $0.27[-0.02,0.58]$ \\
HPFS - Past Five Years & 5.58 & 175 & $<.001$ & $0.84[0.56,1.15]$ \\
HPFS - Past Year & 4.35 & 175 & $<.001$ & $0.66[0.36,0.96]$ \\
TAP (Provoked) & 0.44 & 85 & .659 & $0.10[-0.34,0.53]$ \\
TAP (Unprovoked) & -1.53 & 88 & .131 & $-0.33[-0.75,0.10]$ \\
\hline
\end{tabular}


Supplemental Table 3. Component loadings from the PCA on TAP settings from Study

1.

\begin{tabular}{|c|c|c|c|c|c|c|c|c|}
\hline & \multicolumn{8}{|c|}{ Components } \\
\hline & I & II & III & IV & V & $\mathrm{VI}$ & VII & VIII \\
\hline Eigenvalue & 22.81 & 3.18 & 2.32 & 1.50 & 1.32 & 1.30 & 1.07 & 1.03 \\
\hline $\begin{array}{l}\text { \% Variance } \\
\text { Explained }\end{array}$ & 45.62 & 6.37 & 4.63 & 3.00 & 2.63 & 2.59 & 2.14 & 2.06 \\
\hline Trial 1 - Volume & .66 & -.14 & -.30 & .39 & -.21 & .06 & -.06 & .15 \\
\hline Trial 2 - Volume & .65 & -.48 & -.25 & .14 & .01 & .01 & -.01 & -.06 \\
\hline Trial 3 - Volume & .69 & -.39 & -.24 & .10 & -.03 & .13 & .01 & .01 \\
\hline Trial 4 - Volume & .70 & -.23 & -.14 & -.11 & .17 & .19 & .22 & .07 \\
\hline Trial 5 - Volume & .71 & -.20 & -.26 & .12 & -.13 & .13 & .04 & -.22 \\
\hline Trial 6 - Volume & .76 & -.10 & -.16 & .20 & -.03 & .01 & .09 & -.01 \\
\hline Trial 7 - Volume & .75 & .08 & -.17 & .08 & -.01 & .16 & .15 & -.05 \\
\hline Trial 8 - Volume & .75 & .13 & -.04 & .05 & -.26 & .21 & .19 & .09 \\
\hline Trial 9 - Volume & .68 & .24 & -.14 & .01 & .05 & .25 & .16 & -.03 \\
\hline Trial 10 - Volume & .64 & .35 & -.02 & -.06 & .08 & .44 & -.01 & .12 \\
\hline Trial 11 - Volume & .66 & .36 & -.14 & .03 & -.27 & .04 & -.01 & -.26 \\
\hline Trial 12 - Volume & .70 & .29 & -.04 & .13 & -.28 & .11 & -.16 & -.24 \\
\hline Trial 13 - Volume & .69 & .38 & -.09 & .04 & -.13 & -.02 & -.07 & .14 \\
\hline Trial 14 - Volume & 69 & .12 & .00 & .01 & -.02 & .07 & -.43 & .01 \\
\hline Trial 15 - Volume & .63 & .15 & -.08 & -.24 & .09 & .21 & -.35 & .12 \\
\hline Trial 16 - Volume & .72 & -.16 & .16 & -.11 & .21 & .06 & -.22 & -.26 \\
\hline Trial 17 - Volume & .64 & -.44 & .07 & .03 & .01 & .22 & -.27 & -.05 \\
\hline Trial 18 - Volume & .68 & -.19 & .24 & -.01 & .07 & .12 & -.06 & .11 \\
\hline Trial 19 - Volume & .70 & -.29 & .26 & -.09 & -.07 & .00 & .07 & -.08 \\
\hline Trial 20 - Volume & .66 & -.22 & .21 & -.30 & -.15 & .31 & .10 & .05 \\
\hline Trial 21 - Volume & .75 & -.08 & .07 & -.14 & -.39 & -.12 & .02 & -.03 \\
\hline Trial 22 - Volume & .71 & -.18 & .20 & .21 & -.21 & -.01 & -.13 & .15 \\
\hline Trial 23 - Volume & .73 & .12 & .23 & .19 & -.09 & -.08 & -.10 & .19 \\
\hline Trial 24 - Volume & .68 & .14 & .30 & .29 & .14 & .14 & .11 & .13 \\
\hline Trial 25 - Volume & .58 & .33 & .35 & .11 & .12 & .11 & .01 & .01 \\
\hline Trial 1 - Duration & .62 & -.01 & -.39 & .32 & -.02 & -.10 & -.05 & .22 \\
\hline Trial 2 - Duration & .64 & -.45 & -.33 & .10 & .13 & -.12 & -.04 & -.12 \\
\hline Trial 3 - Duration & .69 & -.28 & -.34 & -.01 & .17 & -.17 & .00 & .06 \\
\hline Trial 4 - Duration & .69 & -.27 & -.14 & -.10 & .26 & -.04 & .21 & .06 \\
\hline Trial 5 - Duration & .73 & -.13 & -.13 & .07 & .17 & -.05 & .09 & -.23 \\
\hline Trial 6 - Duration & .75 & .04 & -.15 & -.01 & .18 & -.07 & .01 & -.01 \\
\hline Trial 7 - Duration & .63 & .20 & -.28 & -.27 & .14 & -.11 & .21 & .06 \\
\hline Trial 8 - Duration & .73 & .10 & -.10 & -.17 & -.10 & -.07 & .06 & .16 \\
\hline Trial 9 - Duration & .61 & .28 & -.19 & -.10 & -.03 & -.25 & .22 & -.14 \\
\hline Trial 10 - Duration & .57 & .42 & -.16 & -.21 & .13 & .11 & .02 & .26 \\
\hline Trial 11 - Duration & .67 & .42 & -.06 & -.06 & .01 & -.15 & .07 & -.16 \\
\hline
\end{tabular}




\begin{tabular}{lllllllll} 
Trial 12 - Duration & .70 & .36 & -.21 & -.16 & -.04 & -.15 & -.11 & -.17 \\
Trial 13 - Duration & .67 & .32 & .05 & -.03 & .07 & -.10 & -.03 & .05 \\
Trial 14 - Duration & .65 & .25 & -.02 & .05 & .17 & -.28 & -.23 & .09 \\
Trial 15 - Duration & .71 & .19 & -.03 & -.19 & .13 & -.21 & -.17 & -.04 \\
Trial 16 - Duration & .71 & -.14 & .17 & -.23 & .25 & .07 & -.06 & .03 \\
Trial 17 - Duration & .66 & -.25 & .19 & .01 & .17 & -.17 & -.19 & -.02 \\
Trial 18 - Duration & .66 & -.18 & .28 & -.11 & .17 & -.11 & .07 & .03 \\
Trial 19 - Duration & .64 & -.29 & .23 & -.14 & -.26 & -.13 & .05 & -.03 \\
Trial 20 - Duration & .60 & -.23 & .22 & -.39 & -.23 & .06 & .13 & .02 \\
Trial 21 - Duration & .75 & -.11 & .15 & -.21 & -.24 & -.21 & .01 & -.09 \\
Trial 22 - Duration & .66 & -.08 & .22 & .02 & -.11 & -.27 & .05 & .30 \\
Trial 23 - Duration & .62 & .03 & .36 & .23 & -.03 & -.30 & .13 & .08 \\
Trial 24 - Duration & .57 & .16 & .38 & .35 & .22 & .00 & .25 & -.02 \\
Trial 25 - Duration & .56 & .21 & .41 & .23 & .14 & .09 & .06 & -.43 \\
\hline
\end{tabular}

Supplemental Table 4: https://osf.io/a2wft/files/ 
Supplemental Table 5. Gender differences in self-reported aggression variables from Study 2, as well as gender differences in TAP scores presented separately by provocation condition $($ Male $=1$, Female $=0) . B P A Q=$ Buss-Perry Aggression Questionnaire, HPFS $=$ History of Physical Fights Scale, TAP = Taylor Aggression Paradigm. 


\begin{tabular}{lllll}
\hline Variable & $t$ & $d f$ & $p$ & $d[95 \% \mathrm{Cl}]$ \\
\hline BPAQ - Physical Aggression & 2.02 & 165 & .046 & $0.31[0.01,0.60]$ \\
BPAQ - Verbal Aggression & 0.91 & 165 & .364 & $0.14[-0.16,0.45]$ \\
HPFS - Past Five Years & 1.45 & 165 & .149 & $0.23[-0.08,0.53]$ \\
HPFS - Past Year & 2.82 & 165 & .010 & $0.44[0.10,0.72]$ \\
TAP (Provoked) & -1.01 & 82 & .315 & $-0.22[-0.67,0.22]$ \\
TAP (Unprovoked) & 0.87 & 81 & .386 & $0.19[-0.25,0.64]$ \\
\hline
\end{tabular}

Supplemental Table 6. Component loadings from the PCA on TAP settings from Study 2.

\begin{tabular}{lccccccccc}
\hline & \multicolumn{10}{c}{ Components } \\
& $\mathrm{I}$ & $\mathrm{II}$ & $\mathrm{III}$ & $\mathrm{IV}$ & $\mathrm{V}$ & $\mathrm{VI}$ & $\mathrm{V}$ II & \multicolumn{1}{l}{ VIII } \\
\hline Eigenvalue & 25.10 & 2.59 & 2.17 & 1.87 & 1.46 & 1.36 & 1.17 & 1.12 \\
\hline $\begin{array}{l}\text { \% Variance } \\
\text { Explained }\end{array}$ & 50.19 & 5.18 & 4.33 & 3.74 & 2.92 & 2.71 & 2.33 & 2.24 \\
\hline Trial 1 - Volume & .58 & -.31 & -.02 & .04 & -.45 & .31 & -.13 & -.15 \\
Trial 2 - Volume & .65 & -.51 & -.08 & .19 & .02 & .03 & -.03 & .00
\end{tabular}




\begin{tabular}{|c|c|c|c|c|c|c|c|c|}
\hline Trial 3 - Volume & .73 & -.37 & -.01 & .18 & .06 & .07 & -.12 & .09 \\
\hline Trial 4 - Volume & .76 & -.39 & .07 & .04 & .07 & -.10 & -.10 & .13 \\
\hline Trial 5 - Volume & .71 & -.35 & .10 & .06 & .10 & -.17 & -.15 & .23 \\
\hline Trial 6 - Volume & .77 & -.24 & .22 & .01 & -.02 & -.25 & -.03 & -.18 \\
\hline Trial 7 - Volume & .77 & -.04 & .19 & -.04 & -.15 & -.38 & .01 & -.12 \\
\hline Trial 8 - Volume & .79 & -.07 & .10 & -.02 & -.14 & -.35 & .00 & -.20 \\
\hline Trial 9 - Volume & .77 & .10 & .11 & -.02 & -.27 & -.04 & -.22 & -.04 \\
\hline Trial 10 - Volume & .77 & .08 & .16 & -.02 & -.26 & .11 & .02 & -.03 \\
\hline Trial 11 - Volume & .72 & .14 & .20 & .14 & -.34 & .01 & .17 & .03 \\
\hline Trial 12 - Volume & .77 & .13 & .25 & .06 & -.28 & -.03 & -.03 & -.0 \\
\hline Trial 13 - Volume & .69 & .10 & .31 & .18 & -.17 & .11 & -.27 & . \\
\hline Trial 14 - Volume & .72 & .02 & .07 & .23 & -.16 & .17 & -.21 & .27 \\
\hline Trial 15 - Volume & .73 & -.02 & .06 & .11 & .10 & .04 & .07 & .1 \\
\hline Trial 16 - Volume & .74 & -.05 & -.24 & -.17 & -.10 & -.18 & .17 & \\
\hline Trial 17 - Volume & .75 & .00 & -.31 & -.08 & -.20 & -.20 & .14 & .2 \\
\hline Trial 18 - Volume & .76 & -.02 & -.22 & -.18 & -.24 & -.14 & .19 & \\
\hline Trial 19 - Volume & .76 & -.04 & -.30 & -.19 & -.02 & .05 & .00 & -.02 \\
\hline Trial 20 - Volume & .73 & -.02 & -.42 & -.12 & -.05 & -.01 & -.09 & $.0 \varepsilon$ \\
\hline Trial 21 - Volume & .77 & .17 & -.29 & -.10 & -.04 & -.15 & -.18 & -.07 \\
\hline Trial 22 - Volume & .77 & .22 & -.19 & .15 & -.02 & -.05 & -.25 & -.09 \\
\hline Trial 23 - Volume & .70 & .23 & -.16 & .24 & .02 & -.13 & -.14 & .07 \\
\hline Trial 24 - Volume & .68 & .24 & -.19 & .44 & -.07 & -.14 & .17 & .00 \\
\hline Trial 25 - Volume & .69 & .22 & .02 & .43 & .01 & .07 & .25 & -.07 \\
\hline Trial 1 - Duration & .58 & -.30 & .04 & -.08 & -.27 & .46 & -.03 & -.26 \\
\hline Trial 2 - Duration & .67 & -.46 & .00 & .15 & .15 & .13 & .14 & -.1 \\
\hline Trial 3 - Duration & .72 & -.32 & .00 & .12 & .25 & .18 & .00 & -.01 \\
\hline Trial 4 - Duration & .72 & -.40 & .08 & .01 & .27 & -.04 & -.09 & -.07 \\
\hline Trial 5 - Duration & .70 & -.24 & .23 & .08 & .31 & -.04 & .03 & .06 \\
\hline Trial 6 - Duration & .76 & -.14 & .23 & -.07 & .07 & -.11 & .06 & -.3 \\
\hline Trial 7 - Duration & .72 & .02 & .20 & -.08 & .11 & -.28 & .11 & -.24 \\
\hline Trial 8 - Duration & .70 & .15 & .31 & -.08 & .32 & -.15 & .02 & .0 \\
\hline Trial 9 - Duration & .63 & .26 & .29 & -.37 & .03 & .08 & -.22 & .0 \\
\hline Trial 10 - Duration & .69 & .24 & .14 & -.17 & .03 & .25 & .09 & -.1 \\
\hline Trial 11 - Duration & .69 & .14 & .20 & -.45 & .08 & .14 & .03 & -.03 \\
\hline Trial 12 - Duration & .65 & .26 & .36 & -.18 & -.01 & -.10 & .12 & .0 \\
\hline Trial 13 - Duration & .65 & .22 & .29 & -.10 & .05 & .13 & .05 & .2 \\
\hline Trial 14 - Duration & .66 & .22 & .17 & -.07 & .13 & .19 & .09 & .2 \\
\hline Trial 15 - Duration & .68 & -.01 & .08 & -.19 & .12 & .27 & .23 & \\
\hline
\end{tabular}




\begin{tabular}{lllllllll} 
Trial 16 - Duration & .70 & -.07 & -.17 & -.12 & .04 & .02 & .25 & .26 \\
Trial 17 - Duration & .68 & -.13 & -.30 & -.09 & -.03 & .02 & .27 & .10 \\
Trial 18 - Duration & .70 & -.05 & -.32 & -.24 & .10 & .19 & .11 & -.10 \\
Trial 19 - Duration & .75 & -.07 & -.22 & -.22 & -.03 & .00 & .02 & -.08 \\
Trial 20 - Duration & .67 & .03 & -.30 & -.12 & .16 & .00 & -.31 & .00 \\
Trial 21 - Duration & .66 & .29 & -.29 & -.10 & .30 & .05 & -.31 & -.06 \\
Trial 22 - Duration & .73 & .30 & -.17 & -.13 & .07 & .05 & .06 & -.30 \\
Trial 23 - Duration & .72 & .34 & -.12 & .14 & .15 & .04 & -.18 & -.06 \\
Trial 24 - Duration & .61 & .31 & -.13 & .48 & .09 & .11 & .07 & -.09 \\
Trial 25 - Duration & .64 & .26 & .01 & .38 & .18 & .12 & .20 & -.18 \\
\hline
\end{tabular}

